\title{
Density of States of a Type-II Superconductor in a High Magnetic Field: Impurity Effects
}

\author{
Saša Dukan ${ }^{a}$ and Zlatko Tešanović $b$ \\ a) Department of Physics and Astronomy, Goucher College, Baltimore, MD 21204 \\ b) Department of Physics and Astronomy, Johns Hopkins University, Baltimore, MD 21218
}

\begin{abstract}
We have calculated the density of states $\mathcal{N}(\omega)$ of a dirty but homogeneous superconductor in a high magnetic field. We assume a dilute concentration of scalar impurities and find how $\mathcal{N}(\omega)$ behaves as one crosses from the weak scattering to the strong scattering limit. At low energies, $\mathcal{N}(\omega) \sim \omega^{2}$ for small values of the impurity concentration and scattering strength. When the disorder becomes stronger than some critical value, a finite density of states is created at the Fermi surface. These results are a consequence of the gapless nature of the quasiparticle excitation spectrum in a high magnetic field.
\end{abstract}

PACS numbers: 72.20.My, 71.45.-d, 71.30.+h 


\section{MOTIVATION}

When an electronic system is placed in an external magnetic field, motion of electrons perpendicular to the field direction is confined to cyclotron orbits. The electronic energies are quantized in the form of a discrete set of Landau levels (LL's) separated by $\hbar \omega_{c}$, where $\omega_{c}=e H / m c$ is the cyclotron frequency. In high magnetic fields and at low temperatures, LL quantization leads to unusual behavior in many-body systems. Perhaps the most vivid example of such behavior is the fractional quantum Hall effect in a two-dimensional (2D) Fermi system subjected to a strong perpendicular magnetic field. There has also been much interest lately in the influence of LL quantization on properties of superconductors in a magnetic field. It was shown by Tešanović et.al. [1], that standard Abrikosov-Gor'kov theory of type-II superconductors which neglects LL quantization, necessarily breaks down at high fields and low temperatures. The inclusion of LL's in the BCS description of the superconducting instability leads to reentrant behavior at high fields (much larger than the semi-classical upper critical field $\left.H_{c 2}(T)\right)$ where the superconductivity is enhanced by a magnetic field [1]. As a consequence of the underlying LL structure, $H_{c 2}(T)$, or rather $T_{c}(H)$, develops oscillations near $H_{c 2}(0)$. Similar types of quantum oscillations, with the same origin, have been predicted in various other measurable quantities which are particularly pronounced in 2D systems [2]. An important theoretical question in this context is the nature of the quasiparticle excitation spectrum at high magnetic fields. It is well known that the existence (or, typically, absence) of low-energy excitations in the superconducting state shapes the behavior of all superconducting thermodynamic and transport properties. The problem of quasiparticle excitations has been studied extensively in the low field limit $\left(H \geq H_{c 1}(T)\right)$ in the context of an isolated vortex line. Scanning tunneling experiments [3] have revealed the structure of bound states in the cores of isolated vortices at low fields, thereby confirming an early prediction of localized midgap states by Caroli, de Gennes and Matricon [4]. Only recently, was the problem of quasiparticle excitations in high magnetic field in the presence of a vortex lattice addressed by a number of groups [5] 8]: At high 
magnetic fields in extreme type-II superconductors vortices are closely packed $\left(H \leq H_{c 2}(T)\right)$ so that the isolated vortex picture from the low-field limit necessarily breaks down. In clean samples at low temperatures quasiparticles can propagate coherently over many unit cells of the vortex lattice. The solution of the BCS problem [5], where the coherent nature of quasiparticle excitations is fully accounted for, points to the qualitatively new nature of the energy spectrum at high fields: at fields near $H_{c 2}$ this spectrum is gapless at a discrete set of points on the Fermi surface. This gapless behavior in 3D systems persists to a surprisingly low magnetic field $\sim 0.5 H_{c 2}$ [9], below which the gaps start opening up, and the system eventually reaches the regime of localized states in the cores of isolated vortices [10]. The gapless character of the excitation spectrum leads to algebraic behavior of various thermodynamic functions in the high field/low temperatures portion of the H$\mathrm{T}$ phase diagram [5]. In particular, it was shown by Dukan and Tešanović 111 that the recent observations of the $\mathrm{dHvA}$-oscillations in the mixed state of A-15 superconductors 12,13 follow from the presence of a small portion of the Fermi surface containing gapless quasiparticle excitations, surrounded by regions where the BCS gap is large. This picture has received further support in the dHvA experiments on $\mathrm{YNi}_{2} \mathrm{~B}_{2} \mathrm{C}$ [14].

In this paper we address the influence of the non-magnetic (scalar) impurities on the gapless superconducting state in high fields, by examining the behavior of the superconducting density of states (DOS) in the presence of disorder. We are interested in a dirty but homogeneous superconductor for which the coherence length $\xi$ is much longer then the effective range $\xi_{i m p}$ of the impurity potential. Under this condition the order parameter in the mixed state is not substantially affected by the impurities and it is assumed to form a perfect triangular vortex lattice. In the opposite, inhomogeneous, limit the impurity potential can pin the vortex making an otherwise perfect vortex lattice disordered. The problem of the superconducting instability and the quasiparticle excitation spectrum in the presence of randomly distributed vortex lines has been addressed so far only by Gedik and Tešanović in the quantum limit, where electrons occupy only the lowest LL. It was demonstrated that the gaplessness of the excitation spectrum found in a pure superconductor is preserved even 
when the positions of the vortex lines are completely random [15].

To understand the effect of disorder on the superconducting properties in the high-field limit near $H_{c 2}(0)$ we start with the normal state in this limit. This problem has been thoroughly investigated in the study of transport properties in high fields [16], where it was found that the electronic scattering with scalar point-like impurities randomly distributed through the sample leads to isotropic broadening by $\delta E=\hbar / 2 \tau$ of the (LL's), where $1 / 2 \tau$ is the scattering rate due to the disorder. As long as the broadening $\delta E \ll \hbar \omega_{c}$, the discreteness of the LL structure is preserved and the effect of the disorder on the superconducting state can be analyzed pertubatively starting from the results for the pure superconductor in high field. We present a Green's function pertubative approach to impurity effects in this regime based on the theory of superconducting alloys of Abrikosov and Gor'kov [17] This is a standard field-theory technique of treating the disorder in the superconducting state, and has been used extensively in the study of anisotropic superconductors such as heavy-fermion systems, p- or d-wave unconventional superconductors [21] or in the study of superfluidity in ${ }^{3} \mathrm{He}$ films [22].

This paper is organized as follows: In Sec. II we describe a simple model of electronic scattering from point-like scalar impurities randomly distributed in the superconducting material. In Sec. III we develop a self-consistent procedure for calculating the electronic self-energies in the framework of the Self-consistent Born (SCBA) approximation. We discuss the behavior of the superconducting density of states within this approximation. In Sec. IV we study the low-energy properties of the density of states in the self-consistent Tmatrix approximation. In Sec. V we discuss the consequences of our results on various experimentally measurable properties of a superconductor in high magnetic field.

\section{DESCRIPTION OF THE MODEL}

We consider a 3-dimensional (3D) BCS-type, weak-coupling electronic system in a high magnetic field. We assume that the system in question is translationally invariant in the 
normal state. The aspects associated with the true band structure of a particular material are not important for the issues that we are planning to address and are not discussed in this work. Nevertheless, all the results presented here can be modified to include band structure effects. For a weakly to moderately interacting system, one uses a simple shortrange attractive BCS model interaction $V\left(\overrightarrow{r_{1}}, \overrightarrow{r_{2}}\right)=-V \delta\left(\overrightarrow{r_{1}}, \overrightarrow{r_{2}}\right)$ between electrons. The dynamical origin of this interaction (i.e. whether mediated by phonons, charge densityfluctuations, etc.) is not important for our present purposes. We assume that the presence of impurities does not effect the effective electron-electron interaction so that it is still of the BCS form. Furthermore, the coupling constant $V$ is taken to be independent of magnetic field. The mean-field (MF) Hamiltonian for this model system is:

$$
\begin{array}{r}
H=\sum_{\alpha, \beta=1,2} \int \Psi_{\alpha}^{\dagger}(\vec{r})\left[\frac{1}{2 m}\left(-i \hbar \nabla+\frac{e}{c} \vec{A}\right)^{2} \delta_{\alpha \beta}+U_{\alpha \beta}(\vec{r})-g \mu_{B} \vec{\sigma} \cdot \vec{H}(\vec{r})-\mu\right] \Psi_{\beta}(\mathbf{r}) d^{3} r+ \\
\int \Delta(\vec{r}) \Psi_{\uparrow}^{\dagger}(\vec{r}) \Psi_{\downarrow}^{\dagger}(\vec{r}) d^{3} r+h . c .
\end{array}
$$

where $\Psi_{\alpha}(\vec{r})$ are the electron field operators for two spin components and $\mu$ is chemical potential. $\vec{A}$ is the vector potential due to the external field $\vec{H}$, which is taken to be uniform everywhere in the system. Term $-g \mu_{B} \vec{\sigma} \cdot \vec{H}$ describes the Zeeman splitting and $U_{\alpha \beta}(\vec{r})=\sum_{j} U_{\alpha \beta}\left(\vec{r}-\vec{R}_{j}^{i m p}\right)$ is the random impurity contribution. $\Delta(\vec{r})=V<\Psi_{\uparrow}^{\dagger}(\vec{r}) \Psi_{\downarrow}^{\dagger}(\vec{r})>$, with $\langle\ldots\rangle$ denoting thermodynamic average, is the superconducting order parameter.

For simplicity we consider a system in which Zeeman splitting is negligible, i.e. $g \approx 0$. The results are straightforwardly generalized to the $g \approx 2$ case [5] 9]. We assume that there are only non-magnetic (scalar) impurities present in the sample so that $U_{\alpha \beta}(\vec{r})$ does not contain any spin-exchange terms. We consider dilute impurity concentrations for which the MF picture presented in this paper is valid. For a dirty but homogeneous superconductor in which the coherence length $\xi$ is much longer than the effective distance $\xi_{\text {imp }}$ over which the impurity potential changes i.e., $\xi / \xi_{i m p} \gg 1$, the superconducting order parameter $\Delta(\vec{r})$ in Eq. (1) is not affected by the impurities apart from its overall magnitude, and forms a perfect triangular Abrikosov lattice [18]. A generic example of such an impurity potential is an infinitely short-range $\delta$-function potential of the form: 


$$
U_{\alpha \beta}(\vec{r})=\sum_{i} U\left(\vec{r}-\vec{R}_{i}\right)=\sum_{i} U_{o} \delta\left(\vec{r}-\vec{R}_{i}\right)
$$

where $\vec{R}_{i}$ is the location of the $i$ th impurity taken to be completely randomly distributed everywhere in the sample. The scalar scattering amplitude $U_{o}$ is assumed to be isotropic.

It was shown in Ref. 5 that the unperturbed part of MF Hamiltonian (1) with $U_{\alpha \beta}(\vec{r})=0$ can be diagonalized in terms of the basis function of the Magnetic Sublattice Representation (MSR) [19], characterized by the quasi-momentum $\vec{q}$ perpendicular to the direction of the magnetic field. The eigenfunctions of this representation in the Landau gauge $\vec{A}=H(-y, 0,0)$ and belonging to the $m$ th Landau level are:

$$
\begin{array}{r}
\phi_{k_{z}, \vec{q}, m}(\vec{r})=\frac{1}{\sqrt{2^{n} n ! \sqrt{\pi} l}} \sqrt{\frac{b_{y}}{L_{x} L_{y} L_{z}}} \exp \left(i k_{z} \zeta\right) \sum_{k} \exp \left(i \frac{\pi b_{x}}{2 a} k^{2}-i k q_{y} b_{y}\right) \\
\exp \left[i\left(q_{x}+\frac{\pi k}{a}\right) x-1 / 2\left(y / l+q_{x} l+\frac{\pi k}{a} l\right)^{2}\right] H_{m}\left(\frac{y}{l}+\left(q_{x}+\frac{\pi k}{a}\right) l\right) .
\end{array}
$$

where $\zeta$ is the spatial coordinate and $k_{z}$ is the momentum along the field direction. $\vec{a}=(a, 0)$ and $\vec{b}=\left(b_{x}, b_{y}\right)$ are the unit vectors of the triangular vortex lattice, $l=\sqrt{\hbar c / e H}$ is the magnetic length and $L_{x} L_{y} L_{z}$ is the volume of the system. $H_{m}(x)$ is the Hermite polynomial of order $m$. Quasimomenta $\vec{q}$ are restricted to the first Magnetic Brillouin Zone (MBZ) defined by vectors $\vec{Q}_{1}=\left(b_{y} / l^{2},-b_{x} / l^{2}\right)$ and $\vec{Q}_{2}=\left(0,2 a / l^{2}\right)$. In the Landau gauge the Abrikosov order parameter can be written as:

$$
\Delta(\vec{r})=\Delta \sum_{n} \exp \left(i \pi \frac{b_{x}}{a} n^{2}\right) \exp \left(i 2 \pi n x / a-(y / l+\pi n l / a)^{2}\right)
$$

where $\Delta$ is the overall BCS amplitude. The above form of the order parameter is taken to be entirely contained in the lowest LL of Cooper charge $2 e$. This is an excellent approximation in the high-field regime [1]. Normal and anomalous Green's functions for the clean superconductor in this representation can be constructed as:

$$
\begin{array}{r}
\mathcal{G}\left(\vec{r}, \overrightarrow{r^{\prime}} ; \omega\right)=\sum_{n, k_{z}, \vec{q}} \phi_{n, k_{z}, \vec{q}}(\vec{r}) \phi_{n, k_{z}, \vec{q}}^{*}\left(\overrightarrow{r^{\prime}}\right) G_{n}\left(k_{z}, \vec{q} ; \omega\right) \\
\mathcal{F}^{\dagger}\left(\vec{r}, \overrightarrow{r^{\prime}} ; \omega\right)=\sum_{n, k_{z}, \vec{q}} \phi_{n,-k_{z},-\vec{q}}^{*}(\vec{r}) \phi_{n, k_{z}, \vec{q}}^{*}\left(\overrightarrow{r^{\prime}}\right) F_{n}^{*}\left(k_{z}, \vec{q} ; \omega\right)
\end{array}
$$


where $\omega=k_{B} T(2 m+1) \pi$ are the electron Matsubara frequencies. In writing (5) we have taken into account only diagonal (in Landau level index $n$ ) contributions to the Green's functions. (5). This is an excellent approximation in high magnetic fields where $\Delta / \hbar \omega_{c} \ll 1$ and number of occupied Landau level $n_{c}$ is not too large. In this situation we can use the diagonal approximation [1], in which the BCS pairs are formed by the electrons belonging to the mutually degenerate Landau levels at the Fermi surface while the contribution from the Landau levels that are separated by $\hbar \omega_{c}$ or more is included in the renormalization of the effective coupling constant $(V \rightarrow \tilde{V}(H, T))$ [9]. In lower fields, where $n_{c}$ is a large number, the off-diagonal terms in (5) should be included on equal footing. It was shown in Ref. 5 by numerically solving the BCS equations that the off-diagonal pairing does not change the qualitative behavior of the superconductor in a magnetic field as long as the magnetic field is larger than some critical field $H_{\text {critical }}(T)$ (estimated to be $\approx 0.5 H_{c 2}$ at $T \approx 0$ in A-15 superconductors): Placed in a high magnetic field and cooled to low temperatures the type-II superconductor has a gapless excitation spectrum. In the diagonal approximation the gapless branches in the spectrum can be found analytically as:

$$
\begin{aligned}
& E_{n}\left(k_{z}, \vec{q}\right)= \pm \sqrt{\epsilon_{n}^{2}\left(k_{z}\right)+\left|\Delta_{n n}(\vec{q})\right|^{2}} \\
& \epsilon_{n}\left(k_{z}\right)=\frac{\hbar^{2} k_{z}^{2}}{2 m}+\hbar \omega_{c}(n+1 / 2)-\mu
\end{aligned}
$$

where $\Delta_{n m}(\vec{q})$ are the matrix elements of the Abrikosov order parameter (雨) in the MSR representation and can be written as:

$$
\Delta_{n m}(\vec{q})=\frac{\Delta}{\sqrt{2}} \frac{(-1)^{m}}{2^{n+m} \sqrt{n ! m !}} \sum_{k} \exp \left(i \pi \frac{b_{x}}{a} k^{2}+2 i k q_{y} b_{y}-\left(q_{x}+\pi k / a\right)^{2} l^{2}\right) H_{n+m}\left[\sqrt{2}\left(q_{x}+\pi k / a\right) l\right]
$$

Once the off-diagonal pairing is included, the excitation spectrum cannot be written in the simple form (6) and a closed analytic expression for the superconducting Green's function cannot be found. Nevertheless, the qualitative behavior of the quasiparticle excitations, characterized by the nodes in the MBZ, remains the same. The main role of the off-diagonal terms in Eqs. (5) for magnetic field strengths such that $H>H_{\text {critical }}$ is to renormalize 
the slopes around the nodes. Once the magnetic field is lowered below $H_{\text {critical }}$, the gaps start opening up at the Fermi surface signaling the crossover to the low-field regime of quasiparticle states localized in the cores of widely separated vortices [10]. In this paper we are interested in how the disorder affects the excitation spectrum in the gapless regime and,in particular, how the DOS behaves in this high-field (and low-temperature) gapless regime of a dirty superconductor. For this purpose, we argue that taking only the diagonal terms in Eqs. (5) will correctly capture the low-energy behavior of DOS for a wide range of magnetic fields (as long as $H>H_{\text {critical }}$ ) while considerably reducing the computational difficulties introduced by the off-diagonal terms.

Proceeding in the frame described above, the "Fourier transformed" (in the quasimomentum space) Green's functions $G_{n}^{0}\left(k_{z}, \vec{q} ; \omega\right)$ and $F_{n}^{0 *}\left(k_{z}, \vec{q} ; \omega\right)$ for the pure superconductor can be easily calculated as:

$$
\begin{aligned}
G_{n}^{0}\left(k_{z}, \vec{q} ; \omega\right) & =\frac{-i \omega-\epsilon_{n}\left(k_{z}\right)}{\omega^{2}+E_{n}^{2}\left(\vec{q}, k_{z}\right)} \\
F_{n}^{* 0}\left(k_{z}, \vec{q} ; \omega\right) & =\frac{\Delta_{n n}^{*}(\vec{q})}{\omega^{2}+E_{n}^{2}\left(\vec{q}, k_{z}\right)}
\end{aligned}
$$

with $E_{n}\left(k_{z}, \vec{q}\right)$ and $\Delta_{n m}(\vec{q})$ given by Eqs. (6) and (7), respectively.

\section{SELF-CONSISTENT BORN APPROXIMATION}

The equations of motion describing the mixed superconducting state in the presence of scalar impurities are:

$$
\begin{array}{r}
{\left[i \omega+\frac{1}{2 m}\left[\nabla_{\vec{r}}-i e \vec{A}(\vec{r})\right]^{2}+\mu-\sum_{i} U\left(\vec{r}-\vec{R}_{i}\right)\right] \mathcal{G}\left(\vec{r}, \overrightarrow{r^{\prime}} ; \omega\right)+\Delta(\vec{r}) \mathcal{F}^{\dagger}\left(\vec{r}, \overrightarrow{r^{\prime}} ; \omega\right)=\delta\left(\vec{r}-\overrightarrow{r^{\prime}}\right),} \\
{\left[-i \omega+\frac{1}{2 m}\left[\nabla_{\vec{r}}+i e \vec{A}(\vec{r})\right]^{2}+\mu-\sum_{i} U\left(\vec{r}-\vec{R}_{i}\right)\right] \mathcal{F}^{\dagger}\left(\vec{r}, \overrightarrow{r^{\prime}} ; \omega\right)-\Delta^{*}(\vec{r}) \mathcal{G}\left(\vec{r}, \vec{r}^{\prime} ; \omega\right)=0}
\end{array}
$$

where normal and anomalous Green's functions incorporate the interactions between the electrons and impurities. We are interested in the quantities $\mathcal{G}$ and $\mathcal{F}^{\dagger}$ averaged over the positions of the impurities. 
In performing the average over the disorder we follow closely the diagrammatic technique developed by Abrikosov and Gor'kov in Ref. [17] for the study of the superconducting alloys in zero field. First, we notice that for a dirty but homogeneous superconductor the quasimomentum $\vec{q}$ is still a good quantum number, since the Abrikosov lattice is not affected by a short-ranged impurity potential (2). Therefore, the Green's functions in (9) can be expanded in terms of a complete set of eigenfunctions (3) as:

$$
\begin{array}{r}
\mathcal{G}\left(\vec{r}, \overrightarrow{r^{\prime}} ; \omega\right)=\sum_{k_{1}} \sum_{k_{2}} \phi_{k_{1}}(\vec{r}) \phi_{k_{2}}^{*}\left(\overrightarrow{r^{\prime}}\right) G\left(k_{1}, k_{2} ; \omega\right) \\
\mathcal{F}^{\dagger}\left(\vec{r}, \overrightarrow{r^{\prime}} ; \omega\right)=\sum_{k_{1}} \sum_{k_{2}} \phi_{-k_{1}}^{*}(\vec{r}) \phi_{k_{2}}^{*}\left(\overrightarrow{r^{\prime}}\right) F^{*}\left(k_{1}, k_{2} ; \omega\right)
\end{array}
$$

where $k \equiv\left(\vec{q}, k_{z}, n\right)$.

The Hamiltonian for the interaction with impurities contains operator products $\Psi \Psi^{\dagger}$. Therefore, when an impurity is inserted into an electron line, two possibilities arise for each of the propagators $\mathcal{G}, \mathcal{F}$ and $\mathcal{F}^{\dagger}$. These possibilities can be written in matrix form:

$$
\hat{\mathcal{G}}\left(x, x^{\prime}\right) \rightarrow \hat{\mathcal{G}}(x, y) \hat{\sigma}_{z} \hat{\mathcal{G}}\left(y, x^{\prime}\right)
$$

with the $2 \times 2$ matrix $\hat{\mathcal{G}}\left(x, x^{\prime}\right)$ defined in Nambu formalism, $\hat{\sigma}_{z}$ the Pauli matrix, and $x \equiv$ $(\vec{r}, \tau)$, where $\tau$ is the imaginary time. Taking into account the expansion (10) this matrix equation can be rewritten in terms of its "Fourier" components as:

$$
\begin{array}{r}
G\left(k_{1}, k_{2} ; \omega\right)=G^{0}\left(k_{1} ; \omega\right) \delta\left(k_{1}-k_{2}\right)+G^{0}\left(k_{1} ; \omega\right) \sum_{k_{3}, \vec{R}_{i}} U_{k_{1} k_{3}}\left(\vec{R}_{i}\right) G\left(k_{3}, k_{2} ; \omega\right) \\
-F^{0}\left(k_{1} ; \omega\right) \sum_{k_{3}, \vec{R}_{i}} U_{-k_{3}-k_{1}}\left(\vec{R}_{i}\right) F^{*}\left(k_{3}, k_{2} ; \omega\right), \\
F^{*}\left(k_{1}, k_{3} ; \omega\right)=F^{0 *}\left(k_{1} ; \omega\right) \delta\left(k_{1}-k_{2}\right)+F^{0 *}\left(k_{1} ; \omega\right) \sum_{k_{2}, \vec{R}_{i}} U_{k_{1} k_{3}}\left(\vec{R}_{i}\right) G\left(k_{3}, k_{2} ; \omega\right) \\
+G^{0}\left(-k_{1} ;-\omega\right) \sum_{k_{2}, \vec{R}_{i}} U_{-k_{3}-k_{1}}\left(\vec{R}_{i}\right) F^{*}\left(k_{3}, k_{2} ; \omega\right)
\end{array}
$$

with a similar equation for the function $F\left(k_{1}, k_{3} ; \omega\right) . \quad U_{k_{1} k_{2}}\left(\vec{R}_{i}\right)$ is the matrix element of the impurity potential $U\left(\vec{r}-\vec{R}_{i}\right)$ between two eigenstates (3). In high magnetic field we can assume that the scattering potential is weak compared to the separation between LL's 
(given by $\hbar \omega_{c}$ ). Under these circumstances electrons scatter into the states belonging to the same LL. Therefore, in solving Eqs. (12) we will neglect the inter-Landau level scattering that becomes important only at much lower fields.

We first solve Eqs. (12) in the Born approximation by summing the diagrams that describe two consecutive electronic scatterings from the same impurity. Since the impurity atoms are randomly distributed through the system, we have to average expressions (12) over the position of each impurity. For a dilute concentration of scatterers with uncorrelated positions, we encounter two types of averages:

$$
\begin{aligned}
& <U_{k_{1} k_{2}}\left(\vec{R}_{i}\right) U_{k_{2} k_{3}}\left(\vec{R}_{i}\right)>_{\vec{R}_{i}}=n_{i} U_{0}^{2} \frac{b_{y}}{L_{x} L_{y} L_{z} \sqrt{\pi} l} \delta_{k_{z 1}, k_{z 3}} \delta_{\vec{q}_{1}, \vec{q}_{3}} \delta_{n_{1}, n_{3}} S_{n_{1} n_{2}}^{N}\left(\vec{q}_{1}-\vec{q}_{2}\right) \\
& <U_{k_{1} k_{2}}\left(\vec{R}_{i}\right) U_{-k_{3}-k_{2}}\left(\vec{R}_{i}\right)>_{\vec{R}_{i}}=n_{i} U_{0}^{2} \frac{b_{y}}{L_{x} L_{y} L_{z} \sqrt{\pi} l} \delta_{k_{z 1}, k_{z 3}} \delta_{\vec{q}_{1}, \vec{q}_{3}} \delta_{n_{1}, n_{3}} S_{n_{1} n_{2}}^{A}\left(\vec{q}_{1}, \vec{q}_{2}\right)
\end{aligned}
$$

where $<\ldots>_{\vec{R}_{i}}$ denotes the average over the impurity positions and $n_{i}$ is the impurity concentration. We find by inspection that $S_{n_{1} n_{2}}^{N}\left(\vec{q}_{1}-\overrightarrow{q_{2}}\right)$ in Eq. (13) is a weak function of quasimomenta $\vec{q}_{1}-\vec{q}_{2}$ and LL indices $n_{1}$ and $n_{2}$. For $n_{1}+n_{2} \geq 5$, to a very good approximation, $S_{n_{1} n_{2}}^{N}(\vec{q})$ is independent of either $\vec{q}$ or $n$. The function $S_{n_{1} n_{2}}^{A}\left(\vec{q}_{1}, \vec{q}_{2}\right)$ in Eq. (14) is obtained as:

$$
S_{n_{1} n_{2}}^{A}\left(\vec{q}_{1}, \vec{q}_{2}\right)=\sqrt{2} \sum_{k=0}^{m i n\left[n_{1}, n_{2}\right]} \frac{(2 k-1) ! !}{(2 k) ! !} f_{(n 1-k)(n 1-k)}\left(\vec{q}_{1}\right) f_{\left(n_{2}-k\right)\left(n_{2}-k\right)}^{*}\left(\vec{q}_{2}\right)
$$

where matrix elements $f_{n n}(\vec{q})=\Delta_{n n}(\vec{q}) / \Delta_{0}$ are calculated in (7) for the order parameter $\Delta(\vec{r})$ from the lowest LL of BCS pairs (丑).

Using the above averages and the expressions (8) we can bring the set of Eqs. (12) to the form:

$$
\begin{gathered}
\left(i \omega-\epsilon_{n}\left(k_{z}\right)-\Sigma^{N}(\omega)\right) G_{n}\left(k_{z}, \vec{q} ; \omega\right)+\left(\Delta_{n n}(\vec{q})+\Sigma_{n n}^{A}(\vec{q} ; \omega)\right) F_{n}^{*}\left(k_{z}, \vec{q} ; \omega\right)=1, \\
\left(i \omega+\epsilon_{n}\left(k_{z}\right)+\Sigma^{N}(-\omega)\right) F_{n}^{*}\left(k_{z}, \vec{q} ; \omega\right)+\left(\Delta_{n n}^{*}(\vec{q})+\Sigma_{n n}^{A *}(\vec{q}, \omega)\right) G_{n}\left(k_{z}, \vec{q} ; \omega\right)=0,
\end{gathered}
$$


The diagonal (normal) self-energy $\Sigma^{N}(\omega)$ and off-diagonal (anomalous) self-energy $\Sigma_{n n}^{A}(\vec{q} ; \omega)$ can be expressed as:

$$
\begin{array}{r}
\Sigma^{N}(\omega)=n_{i} U_{0}^{2} \frac{b_{y}}{L_{x} L_{y} L_{z} \sqrt{\pi} l} \sum_{m, k_{z}, \vec{k}} G_{m}\left(k_{z}, \vec{k} ; \omega\right) \\
\Sigma_{n n}^{A}(\vec{q} ; \omega)=n_{i} U_{0}^{2} \frac{b_{y}}{L_{x} L_{y} L_{z} \sqrt{\pi} l} \sum_{m, k_{z}, \vec{k}} S_{n m}^{A}(\vec{q}, \vec{k}) F_{m}\left(k_{z}, \vec{k} ; \omega\right)
\end{array}
$$

where the functions $G_{m}\left(k_{z}, \vec{k} ; \omega\right)$ and $F_{m}\left(k_{z}, \vec{k} ; \omega\right)$ are found as a solution of Eqs. (16) and can be written in the form:

$$
\begin{aligned}
G_{m}\left(k_{z}, \vec{k} ; \omega\right) & =\frac{-i \tilde{\omega}-\epsilon_{m}\left(k_{z}\right)}{\tilde{\omega}^{2}+\epsilon_{m}^{2}\left(k_{z}\right)+\left|\tilde{\Delta}_{m m}(\vec{k})\right|^{2}} \\
F_{m}\left(k_{z}, \vec{k} ; \omega\right) & =\frac{\tilde{\Delta}_{m m}(\vec{k})}{\tilde{\omega}^{2}+\epsilon_{m}^{2}\left(k_{z}\right)+\left|\tilde{\Delta}_{m m}(\vec{k})\right|^{2}}
\end{aligned}
$$

with

$$
i \tilde{\omega} \equiv i \omega-\Sigma^{N}(\omega), \quad \tilde{\Delta}_{m m}(\vec{k}) \equiv \Delta_{m m}(\vec{k})+\Sigma_{m m}^{A}(\vec{k} ; \omega)
$$

The sums over $k_{z}$ in Eqs. (17) can be readily done so that Eqs. (19) become self-consistent equations for the self-energies:

$$
\tilde{\omega}=\omega+\Gamma_{0} \frac{1}{N(0)} \sum_{\nu=0}^{n_{c}} \frac{m}{4 \pi^{3} k_{F \nu}} \int d \vec{k} \frac{\tilde{\omega}}{\sqrt{\tilde{\omega}^{2}+\left|\tilde{\Delta}_{\nu \nu}(\vec{k})\right|^{2}}}
$$

and

$$
\begin{aligned}
\tilde{\Delta}_{n n}(\vec{q})=\Delta_{n n}(\vec{q}) & +\frac{\Gamma_{0}}{N(0)} \sum_{k=0}^{n} \frac{\sqrt{2}(2 k-1) ! !}{(2 k) ! !} f_{(n-k)(n-k)}(\vec{q}) \\
& \times \sum_{\nu=k}^{n_{c}} \frac{m}{4 \pi^{3} k_{F \nu}} \int d \vec{k} \frac{f_{(\nu-k)(\nu-k)}^{*}(\vec{k}) \tilde{\Delta}_{\nu \nu}(\vec{k})}{\sqrt{\tilde{\omega}^{2}+\left|\tilde{\Delta}_{\nu \nu}(\vec{k})\right|^{2}}}
\end{aligned}
$$

where $N(0)$ is the density of states at the Fermi level of the normal metal in zero field. $\Gamma_{0}=\pi n_{i} U_{0}^{2} N(0)$ is the scattering rate due to the disorder (defined in zero field). It is assumed that $\Gamma_{0} / E_{F} \ll 1$ within the Born approximation.

The exact self-consistent solution of Eqs. (20) and (21) is extremely difficult to obtain due to the coupling of matrix elements $\Delta_{n n}(\vec{q})$ with $\Delta_{(n-k)(n-k)}(\vec{q})$ in Eq. (21). Nevertheless, 
this problem can be simplified if we notice that the behavior of $\Delta_{n n}(\vec{q})$ for different LL indices is very similar around the gapless points in Eqs. (6) and differs considerably only in the regions in the MBZ that are gapped by large $\Delta$. We are primarily interested in the low-energy behavior of the density of states and low-temperature thermodynamic properties of a dirty superconductor in a high magnetic field. These properties are governed by the quasiparticle excitations around nodes in $\Delta_{n n}(\vec{q})$. Therefore, retaining only the $k=0$ term in Eq. (21) represents a reasonable approximation in solving Eqs. (20) and (21). Furthermore, we have demonstrated numerically that the sum of $k \neq 0$ terms in (21) is $\leq 10 \%$ of the $k=0$ term for small frequencies, while it is negligible for higher frequencies (of order $\Delta$ ). Within this approximation $\tilde{\Delta}_{n n}(\vec{q})=\tilde{\Delta} f_{n n}(\vec{q})$ so that Eqs. (20) and (21) are combined as

$$
u=\frac{\omega}{\Delta}+\zeta \frac{1}{N(0)} \sum_{n=0}^{n_{c}} \frac{m}{4 \pi^{3} k_{F n}} \int d \vec{k} \frac{u\left(1-\sqrt{2}\left|f_{n n}(\vec{k})\right|^{2}\right)}{\sqrt{u^{2}+\left|f_{n n}(\vec{k})\right|^{2}}}
$$

where $u=\tilde{\omega} / \tilde{\Delta}$ and $\zeta=\Gamma_{0} / \Delta$. The amplitude $\Delta \equiv \Delta(H, T, \Gamma)$ has to be determined from the self-consistent equation:

$$
\Delta(\vec{r})=V T \sum_{\omega} F(\vec{r}, \vec{r} ; \omega)
$$

If we take the order parameter $\Delta(\vec{r})$ entirely in the lowest Landau level for the Cooper pair, Eq. (23) can be rewritten as

$$
\Delta=\tilde{V} T \frac{\sqrt{2} b_{y}}{\sqrt{\pi} l L_{x} l_{y} L_{z}} \sum_{\omega} \sum_{k_{z} \vec{q} n} \frac{f_{n n}^{*}(\vec{q}) \tilde{\Delta}_{n n}(\vec{q})}{\tilde{\omega}^{2}+\varepsilon\left(k_{z}\right)^{2}+\left|\tilde{\Delta}_{n n}(\vec{q})\right|^{2}}
$$

where $\tilde{V}(H, T)$ is the BCS pairing interaction that is, in general, renormalized by off-diagonal terms due to the coupling of electrons from LL's separated by $\hbar \omega_{c}$ or more [9]. One can think of $\tilde{V}(H, T)$ as being chosen to reproduce the true self-consistent $\Delta(H, T)$ in a formalism that keeps only the diagonal terms. Its explicit form is easily computed in the $\Delta / \hbar \omega_{c} \ll 1$ regime [9]. Note that, once disorder is included, $\tilde{V}(H, T)$ itself has to be recomputed selfconsistently. Here we ignore this complication on the grounds that a modest sacrifice in quantitative accuracy (for $\Delta / \hbar \omega_{c}<1$ ) is justified in the face of overwhelming numerical difficulty in determining self-consistent $\Delta(H, T)$ in presence of disorder. 
The set of Eqs. (22) and (24) completely describes the effect of disorder on the superconducting state within the Born approximation and enables us to calculate various physical quantities. The superconducting density of states in the presence of impurities is defined as

$$
\mathcal{N}_{s}(\omega)=-\frac{1}{\pi} \Im m \int d \vec{r} \mathcal{G}(\vec{r}, \vec{r} ; i \omega)=-\left.\frac{1}{\pi L_{x} L_{y} L_{z}} \Im m \sum_{n, k_{z}, \vec{q}} G_{n}\left(k_{z}, \vec{q} ; i \omega\right)\right|_{i \omega=\omega+i \delta}
$$

where $\left.G\left(k_{z}, \vec{q} ; i \omega\right)\right|_{i \omega=\omega+i \delta}$ is given by expression (18) in which the analytic continuation to real frequencies is performed. Equation (22) is an implicit equation from which $u=u[\omega / \Delta]$ is to be calculated. Once $u$ is known, the density of states (25) can be obtained as

$$
\mathcal{N}_{s}(\omega) / N(0)=\frac{1}{N(0)} \Im m \sum_{n=0}^{n_{c}} \frac{m}{4 \pi^{3} k_{F n}} \int d \vec{q} \frac{u}{\sqrt{\left|f_{n n}(\vec{q})\right|^{2}-u^{2}}}
$$

In Fig. 1 we plot $\mathcal{N}_{s}(\omega) / N(0)$ for several values of the parameter $\zeta=\Gamma_{0} / \Delta$ when $n_{c}=10$. Two kinds of behavior are present: For $\zeta \leq 0.9$ the superconducting density of states vanishes at the Fermi level and $\mathcal{N}_{s}(\omega) \sim \omega^{2}$ for small $\omega$. This is the same behavior as one finds in a pure system, just that the coefficient in front of $\omega^{2}$ is increased from its clean system value. When $\zeta>0.9$, a finite density of states is created at the Fermi level, although of course it is smaller than in the normal state. In this regime $\mathcal{N}_{s}(\omega) \sim \mathcal{N}_{s}(0)+$ const. $\times \omega$ for small $\omega$. At

higher energies, we observe that the peak in the density of states located at $\omega / \Delta \approx 1 / \sqrt{2}$ in the clean system is reduced and broadened by disorder. As the impurity concentration (measured by the parameter $\zeta$ ) increases, the peak eventually disapeares. Note, though, that our calculation might be less accurate at the higher energies (of the order $\Delta$ ) due to the number of approximations that are, as explained above, strictly applicable only at the low energies. Furthermore, the true behavior of the peak can be investigated only if $\Delta=\Delta(\Gamma, T)$ is found from the self-consistent equation (24).

\section{T-MATRIX APPROXIMATION}

The self-energies $\Sigma_{n n}^{N}(\vec{q} ; \omega)$ and $\Sigma_{n n}^{A}(\vec{q} ; \omega)$ of the superconducting system obeying Eqs. (16) are closely related to the diagonal (with respect to the Magnetic Translation Group basis) T-matrix elements in a single-site approximation as 


$$
\begin{array}{r}
\Sigma_{n n}^{N}(\vec{k} ; \omega)=n_{i}<T^{11}(k, k ; \omega)>_{\vec{R}_{i}} \\
\Sigma_{n n}^{A}(\vec{k} ; \omega)=-n_{i}<T^{12}(k, k ; \omega)>_{\vec{R}_{i}}
\end{array}
$$

where $T^{i j}\left(k_{1}, k_{2} ; \omega\right)$ are the coefficients in the T-matrix expansion over the complete set of eigenstates $k \equiv\left(k_{z}, \vec{k}, n\right)$

$$
\begin{array}{r}
\mathcal{T}^{11}\left(\vec{r}, \vec{r}^{\prime} ; \omega\right)=\sum_{k_{1}, k_{2}} \phi_{k_{1}}(\vec{r}) \phi_{k_{2}}^{*}\left(\vec{r}^{\prime}\right) T^{11}\left(k_{1}, k_{2} ; \omega\right) \\
\mathcal{T}^{12}\left(\vec{r}, \vec{r}^{\prime} ; \omega\right)=\sum_{k_{1}, k_{2}} \phi_{-k_{1}}(\vec{r}) \phi_{k_{2}}\left(\vec{r}^{\prime}\right) T^{12}\left(k_{1}, k_{2} ; \omega\right) .
\end{array}
$$

The $2 \times 2$ T-matrix $\hat{\mathcal{T}}\left(\vec{r}, \vec{r}^{\prime} ; \omega\right)$ obeys the Lippmann-Schwinger equations

$$
\hat{\mathcal{T}}\left(\vec{r}, \vec{r}^{\prime} ; \omega\right)=U(\vec{r}) \delta\left(\vec{r}-\vec{r}^{\prime}\right) \hat{\sigma}_{z}+\int d \vec{r}_{1} U(\vec{r}) \hat{\sigma}_{z} \hat{\mathcal{G}}\left(\vec{r}, \vec{r}_{1} ; \omega\right) \hat{\mathcal{T}}\left(\vec{r}_{1}, \vec{r}^{\prime} ; \omega\right)
$$

where $\hat{\mathcal{G}}$ matrix elements are given by (10) and $U(\vec{r})$ is the impurity potential (2). As in SCBA, we neglect the inter-Landau level scattering on the basis that in high magnetic field the scattering potential is much weaker than $\hbar \omega_{c}$. After averaging over the impurity position, Eqs. (29) for the dirty but homogeneous superconductor reduce to

$$
\begin{array}{r}
T_{n n}^{11}\left(k_{z}, \vec{k} ; \omega\right)=U_{0}+U_{0} \frac{b_{y}}{L_{x} L_{y} L_{z} \sqrt{\pi} l} \sum_{q_{z}, \vec{q}, m} G_{m}\left(q_{z}, \vec{q} ; \omega\right) T_{m n}^{11}\left(q_{z}, \vec{q} ; k_{z}, \vec{k} ; \omega\right) \\
+U_{0} \frac{b_{y}}{l_{x} L_{y} L_{z} \sqrt{\pi} l} \sum_{q_{z}, \vec{q}, m} S_{n m}^{A}(\vec{k}, \vec{q}) F_{m}\left(q_{z}, \vec{q} ; \omega\right) T_{m n}^{21}\left(q_{z}, \vec{q} ; k_{z}, \vec{k} ; \omega\right)
\end{array}
$$

and

$$
\begin{aligned}
T_{n n}^{21}\left(k_{z}, \vec{k} ; \omega\right)=- & U_{0} \frac{b_{y}}{L_{x} L_{y} L_{z} \sqrt{\pi} l} \sum_{q_{z}, \vec{q}, m} S_{n m}^{A *}(\vec{k}, \vec{q}) F_{m}^{*}\left(q_{z}, \vec{q} ; \omega\right) T_{m n}^{11}\left(q_{z}, \vec{q} ; k_{z}, \vec{k} ; \omega\right) \\
& \left.+U_{0} \frac{b_{y}}{L_{x} L_{y} L_{z} \sqrt{\pi} l} \sum_{q_{z}, \vec{q}, m} G_{m}\left(-q_{z},-\vec{q} ;-\omega\right) T_{m n}^{21}\left(q_{z}, \vec{q} ; k_{z}, \vec{k} ; \omega\right)\right)
\end{aligned}
$$

where $G_{m}\left(q_{z}, \vec{q} ; \omega\right)$ and $F_{m}\left(q_{z}, \vec{q} ; \omega\right)$ are written in the form (18) and $S_{n m}^{A}(\vec{k}, \vec{q})$ is given by formula (15). Around the gapless points of the excitation spectrum (6) the second term in equation (30) is very small compared to the first term. This can be deduced if one inspects expressions (15) and (31) around the nodes of the excitation spectrum (6) in the MBZ. Keeping this conclusion in mind, equations (30) and (31) can be solved as 


$$
T^{11}(\omega)=\frac{\frac{b_{y}}{L_{x} L_{y} L_{z} \sqrt{\pi} l} \sum_{q_{z}, \vec{q}, m} G_{m}\left(q_{z}, \vec{q}, m\right)}{1 / U_{0}^{2}-\left[\frac{b_{y}}{L_{x} L_{y} L_{z} \sqrt{\pi} l} \sum_{q_{z}, \vec{q}, m} G_{m}\left(q_{z}, \vec{q}, m\right)\right]^{2}}
$$

and

$$
T_{n n}^{21}(\vec{k} ; \omega)=-\frac{\frac{\sqrt{2} b_{y}}{L_{x} L_{y} L_{z} \sqrt{\pi} l} f_{n n}^{*}(\vec{k}) \sum_{q_{z}, \vec{q}, m} f_{m m}(\vec{q}) F_{m}^{*}\left(q_{z}, \vec{q} ; \omega\right)}{1 / U_{0}^{2}-\left[\frac{b_{y}}{L_{x} L_{y} L_{z} \sqrt{\pi} l} \sum_{q_{z}, \vec{q}, m} G_{m}\left(q_{z}, \vec{q}, m\right)\right]^{2}}
$$

where we used the explicit form (15) for $S_{n m}^{A}(\vec{k}, \vec{q})$ (taking only the $k=0$ term in Eq. (15), see the discussion in previous section). With the help of definitions (19) and (27), the set of equations (32) and (33) can be brought into the form

$$
u=\frac{\omega}{\Delta}+\zeta \frac{\sum_{n} \frac{m}{4 \pi^{3} k_{F n} N(0)} \int d \vec{q}\left(1-\sqrt{2}\left|f_{n n}(\vec{q})\right|^{2}\right) u / \sqrt{u^{2}+\left|f_{n n}(\vec{q})\right|^{2}}}{c^{2}+\left[\sum_{n} \frac{m}{4 \pi^{3} k_{F n} N(0)} \int d \vec{q} u / \sqrt{u^{2}+\left|f_{n n}(\vec{q})\right|^{2}}\right]^{2}}
$$

where $\zeta=\Gamma / \Delta$ and $u=\tilde{\omega} / \tilde{\Delta}$.

Disorder is characterized with two parameters: $\Gamma=n_{i} / N(0) \pi=\left(n_{i} / n\right) E_{F}$, which measures the concentration of impurities $n_{i}$ relative to the electron density $n$, and $c=$ $1 / \pi N(0) U_{0}$, which measures the strength of the scattering potential. The normal state inverse scattering rate $1 / 2 \tau$ is found by taking $f_{n n}(\vec{q})=0$ in (34) and letting $\omega \rightarrow 0$. This procedure yields $1 / 2 \tau=\Gamma /\left(1+c^{2}\right)$, the result first obtained in Ref. 17 in the study of the transport properties of normal metals in a high magnetic field. The weak scattering limit is approached when $c^{2}$ is much larger than the second term in the denominator of expression (34), while the strong scattering limit is achieved when $c^{2}=0$. In the strong scattering limit, the approximation in which the inter-Landau level scattering is neglected eventually becomes unphysical, unless the magnetic field is so high that only the lowest LL is occupied.

As in previous section, the superconducting density of states in the presence of impurities is found from Eq. (26) once the solution $u=u[\omega / \Delta]$ of Eq. (34) is found. Figures 2, 3 and 4 show how the superconducting density of states $\mathcal{N}_{s}(\omega) / N(0)$ behaves as a function of the energy parameter $\omega / \Delta$ as one crosses from a weak scattering limit, $c=1.0$, (Fig. 2) to a strong scattering limit, $c=0.0$, (Fig. 4). For each value of $c$ (measuring the scattering strength) we present how the density of states changes as the impurity concentration, i.e., 
parameter $\zeta$ increases. There are two types of behavior present in figures. When $\zeta<c^{2}$ the density of states vanishes at the Fermi level with $\mathcal{N}_{s}(\omega) \sim \omega^{2}$ for small $\omega$, the coefficient in front of $\omega^{2}$ being increased from the clean system value. When $\zeta \approx c^{2}$, the density of states still vanishes at the Fermi level, but $\mathcal{N}_{s}(\omega) \sim \omega$ for small $\omega$. Further increase of the concentration $n_{i}$ such that $\zeta>c^{2}$ creates a finite density of states $\mathcal{N}_{s}(0)$ at the Fermi level. In the strong scattering limit, $c \ll 1$, the superconducting density of states is finite at the Fermi level for any non-zero concentration of impurities. In this limit, $\mathcal{N}_{s}(0) / N(0) \approx 2(\gamma / \Delta)$ where $\gamma=\Delta \sqrt{\zeta / 2}$ for $\zeta \ll 1$. Furthermore, below $\omega / \Delta=\sqrt{\zeta}$ in the strong scattering limit, a peak is observed centered at zero energy. This peak suggests formation of a quasi-bound, resonant state which is analog of a Shiba state formed in the energy gap of a conventional s-wave superconductor, as a result of multiple scattering off a magnetic impurity [20]. As one moves away from the strong scattering limit, this zero-energy peak disappears. At higher energies, we find a similar behavior of the density of states to the one observed in the Born approximation of the previous section: The peak, located at $\omega / \Delta \approx 1 / \sqrt{2}$ in the clean system, is reduced and broadened as the impurity concentration (measured by parameter $\zeta$ ) increases. Also, this peak is slightly shifted to higher $\omega / \Delta$ as $\zeta$ increases, suggesting a stronger reduction in BCS amplitude $\Delta=\Delta(T, \Gamma)$ than what is found in the Born approximation.

\section{CONCLUSIONS}

In this paper we have analyzed the influence of a dilute static disorder on superconducting properties in a high magnetic field. We considered a dirty but homogeneous superconductor for which the order parameter $\Delta(\vec{r})$ is not influenced by the presence of the impurities and still forms the perfect Abrikosov triangular lattice. We considered the weak-scattering limit within a self-consistent Born approximation while the strong-scattering limit was treated within a T-matrix approximation for superconducting self-energies.

We found that for small impurity concentrations and weak scattering potentials the 
superconducting density of states behaves as $E^{2}$ for small energies $E$, the same behavior as that found for the pure superconductor in a magnetic field [5]. When disorder becomes stronger than some critical value, a finite density of states (but still smaller then the normal state value) is created at the Fermi level. The finite superconducting density of states at the Fermi level signals the broadening of gapless points into gapless regions in the MBZ. It is interesting to mention that this behavior is similar to that of dirty superfluid ${ }^{3}$ He films [22], and somewhat similar to the behavior of the density of states in anisotropic heavy-fermion superconductors [21].

The experimental property of a superconductor in which the absence of a quasiparticle gap over some region of the Fermi surface will be most obviously felt is the specific heat. In a clean system in high magnetic field at low temperatures $c_{v} \sim A T^{3}$, where $A$ is the field dependent coefficient [5]. In a dirty but homogeneous superconductor, instead of the $T^{3}$ law one finds linear behavior at low temperatures with the coefficient reduced by the factor $\sim 2(\gamma / \Delta)$ from the normal state value. Detailed measurements of heat capacity at very low temperatures and high magnetic fields are not yet found in the literature. We propose a class of A-15 superconductors as good candidates in which the linear temperature law of heat capacity at high magnetic field can be discovered. These systems have experimentally accessible upper critical fields and are clear examples of materials for which the LL quantization in high fields plays an important role 11 13.

This work has been supported in part by the NSF Grant No. DMR-9415549. 


\section{REFERENCES}

[1] Zlatko Tešanović, M. Rasolt and Li Xing, Phys. Rev. Lett. 63, 2425 (1989); Zlatko Tešanović, M. Rasolt and L. Xing, Phys. Rev. B 43, 288 (1991); M. Rasolt and Z. Tešanović, Rev. Mod. Phys. 64, 709 (1992).

[2] T. Maniv, A. I. Rom, I. D. Vagner and P. Wyder, Phys. Rev. B 46, 8360 (1992).

[3] H. F. Hess, R. B. Robinson, R. C. Dynes, J. M. Valles and J. V. Waszczak, Phys. Rev. Lett. 62, 214 (1989); H. F. Hess, R. B. Robinson and J. V. Waszczak, Phys. Rev. Lett. 64, $2711(1990)$.

[4] C. Caroli, P. G. de Gennes and J. Matricon, Phys. Lett. 9, 307 (1964).

[5] S. Dukan, A. V. Andreev and Z. Tešanović, Physica C 183, 355 (1991); S. Dukan and Z. Tešanović, Phys. Rev. B 49, 13017 (1994).

[6] M. J. Stephen, Phys. Rev. B 45, 5481 (1992).

[7] H. Akera, A. H. MacDonald, S. M. Girvin and M. R. Norman, Phys. Rev. Lett. 67, 2375 (1991); A. H. MacDonald, H. Akera and M. R. Norman, Phys. Rev. B 45, 10147 (1992); M. R. Norman, H. Akera and A. H. MacDonald, Physica C 196, 43 (1992).

[8] G. M. Bruun, V. Nikos Nicopoulos and N. F. Johnson, supr-con/9608004 preprint (1996). See also P. Miller and B. L. Gyorffy, J. Phys. Cond. Matter 7, 5579 (1995) for a different but related approach.

[9] S. Dukan, Ph. D. Thesis, Johns Hopkins University (1995).

[10] M. R. Norman, A. H. MacDonald and H. Akera, Phys. Rev. B 51, 5927 (1995).

[11] S. Dukan and Z. Tešanović, Phys. Rev. Lett. 74, 2311 (1995).

[12] R. Corcoran, N. Harrison, S. M Hayden, P. Meeson, M. Springford and P. J. van der Wel, Phys. Rev. Lett. 72, 701 (1994). 
[13] N. Harrison, S. M. Hayden, P. Meeson, M. Springford, P. J. van der Wel and A. A. Menovsky, Phys. Rev. B 50, 4208 (1994); R. Corcoran, N. Harrison, C. J. Haworth, S. M. Hayden, P. Meeson, M. Springford and P. J. van der Wel, Physica B 206-207, 534 (1995).

[14] G. Goll, M. Heinecke, K. Winzer and P. Wyder, Phys. Rev. B 53, R8871 (1996).

[15] Z. Gedik and Z. Tešanović, Phys. Rev. B 52, 527 (1995).

[16] A. A. Abrikosov, Zh. Eksp. Teor. Fiz. 56, 1391 (1969) [Sov. Phys.-JETP 29, 746 (1969).

[17] A. A. Abrikosov and L. P. Gorkov, Zh. Eksp. Teor. Fiz. 39, 866 (1961) [Sov. Phys.-JETP 12, 1242 (1961).

[18] A. A. Abrikosov, JETP 51174 (1957); Zh. Exp. Ter. Fiz. 32, 1442 (1957).

[19] Y. A. Bychkov and E. I. Rashba, Zh. Eksp. Teor. Fiz. 85, 1826 (1983).

[20] H. Shiba, Prog. Theor. Phys. 40, 435 (1968).

[21] P. J. Hirschfeld and P. Wölfle, Phys. Rev. B 37, 83 (1988).

[22] Z. Tešanović and O. T. Valls, Phys. Rev. B 34, 7610 (1986). 


\section{FIGURES}

FIG. 1. Quasiparticle density of states $\mathcal{N}_{s}(\omega) / N(0)$ vs reduced energy $\omega / \Delta$ in the Born approximation as a function of parameter $\zeta=\Gamma_{0} / \Delta$.

FIG. 2. Quasiparticle density of states $\mathcal{N}_{s}(\omega) / N(0)$ vs reduced energy $\omega / \Delta$ when $c=1.0$, as a function of the impurity concentration parameter $\zeta=\Gamma / \Delta$.

FIG. 3. Quasiparticle density of states $\mathcal{N}_{s}(\omega) / N(0)$ vs reduced energy $\omega / \Delta$ when $c=0.5$, as a function of the impurity concentration parameter $\zeta=\Gamma / \Delta$.

FIG. 4. Quasiparticle density of states $\mathcal{N}_{s}(\omega) / N(0)$ vs reduced energy $\omega / \Delta$ when $c=0.0$, as a function of the impurity concentration parameter $\zeta=\Gamma / \Delta$. 







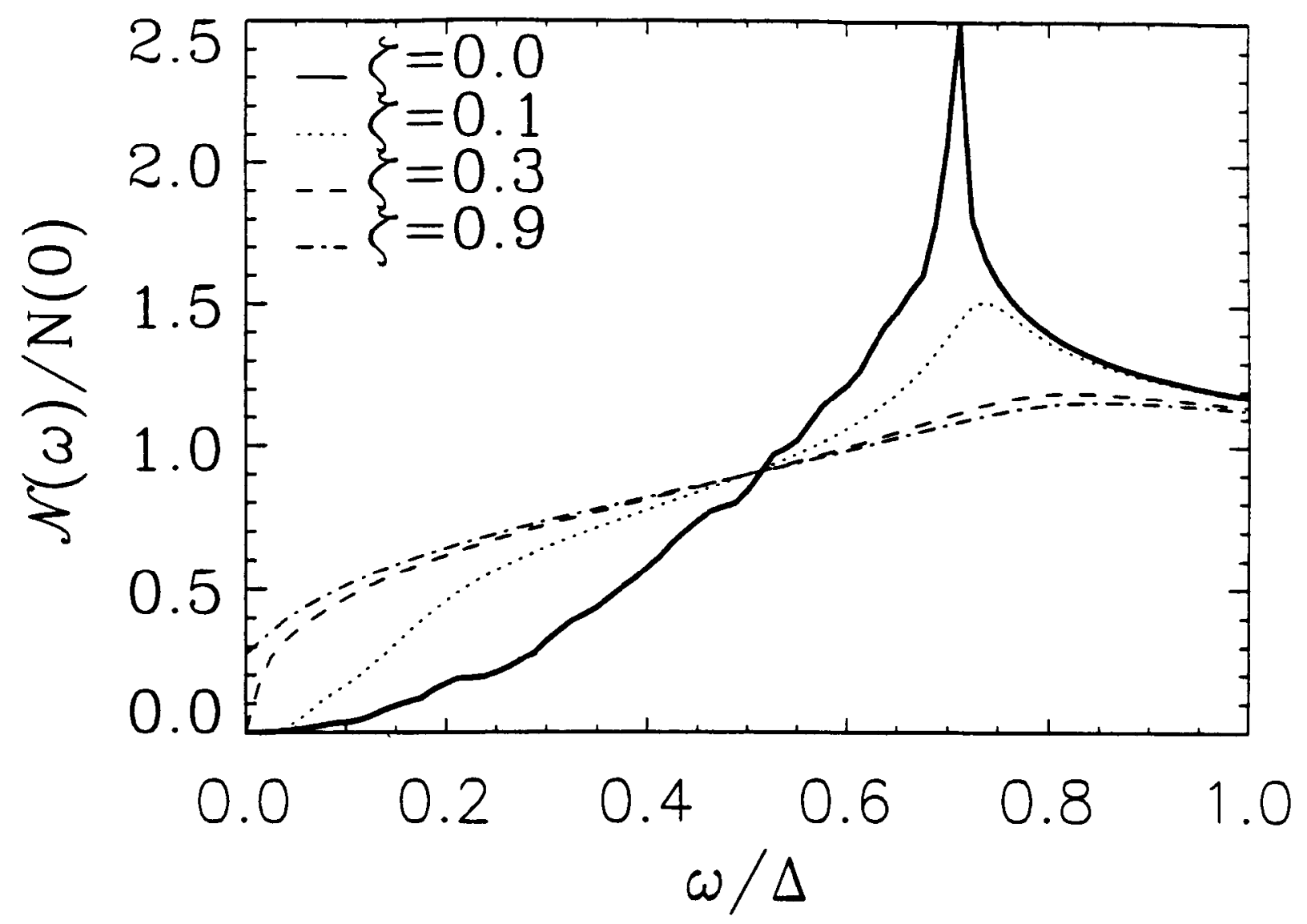




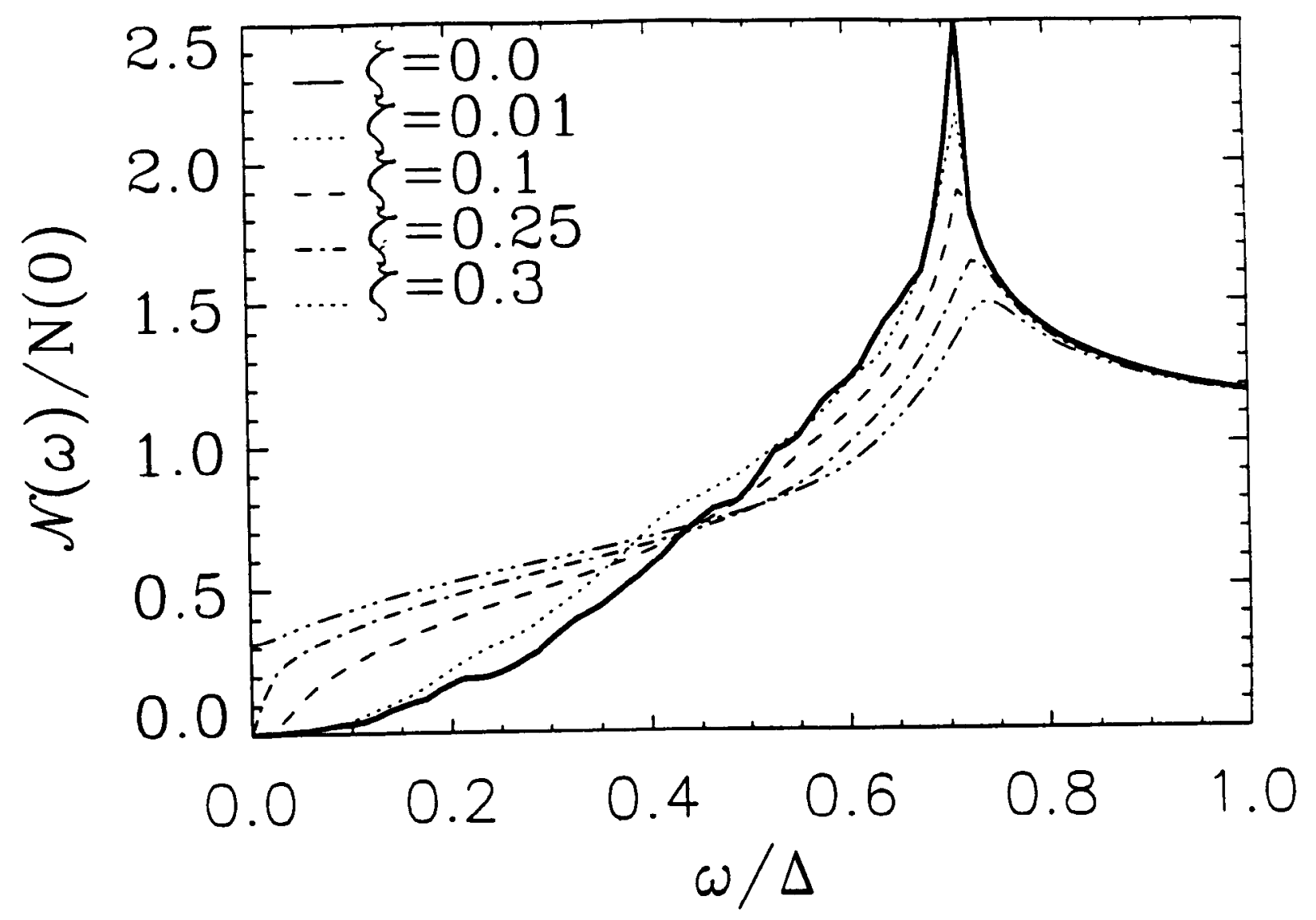




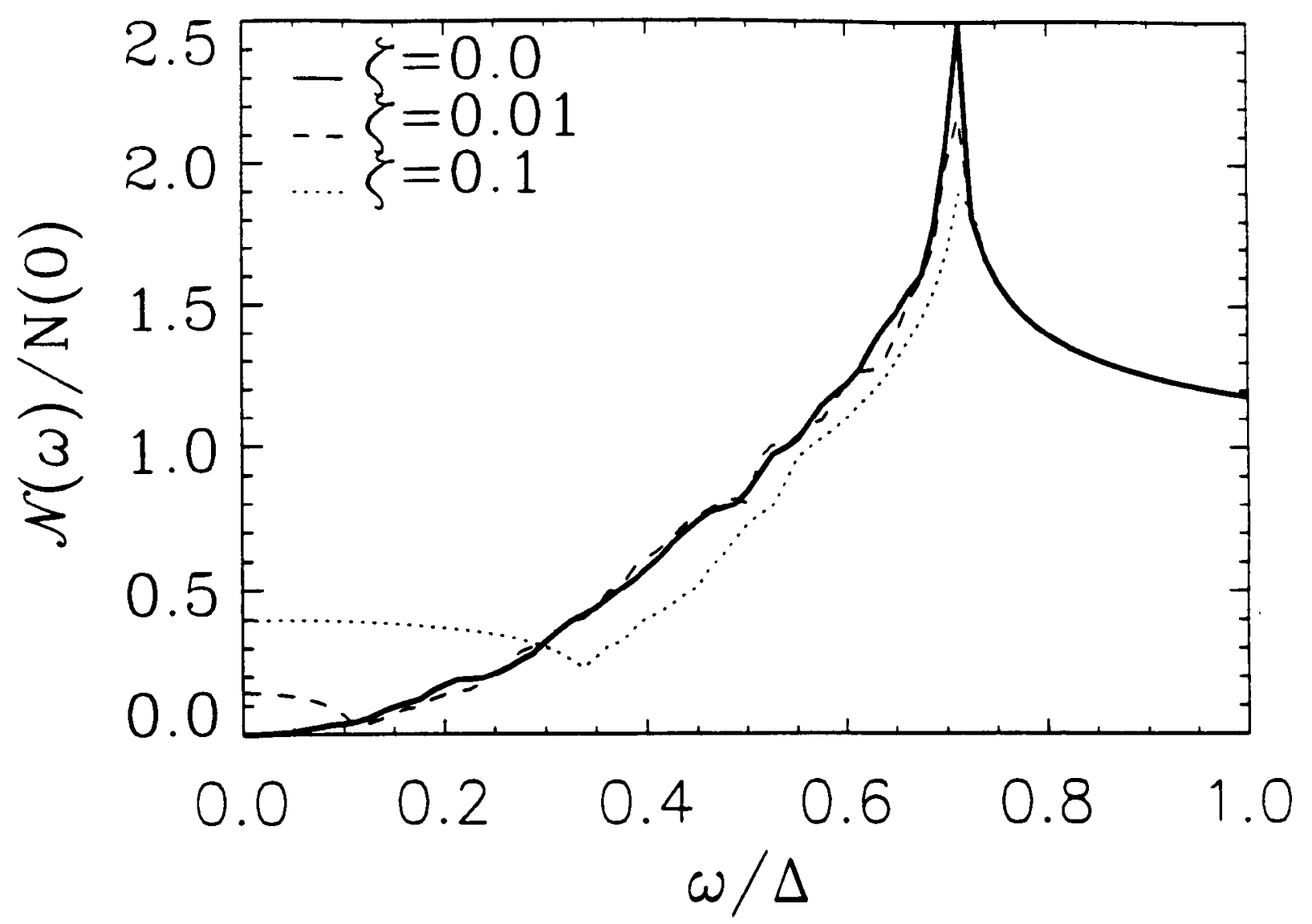

